\title{
Evaluación de la eficacia de la terapia ablativa con argón plasma en el esófago de Barrett con displasia: seguimiento prospectivo no menor a un año
}

\section{Evaluation of the Efficacy of Argon Plasma Treatment of Barrett's Esophagus with Dysplasia: Prospective Follow-up Evaluation not less than one year}

\author{
Rodrigo Castaño, MD, ${ }^{1}$ Oscar Álvarez, MD, ${ }^{2}$ Amy Piñeres, MD, ${ }^{3}$ Mario H Ruiz, MD, ${ }^{4}$ Andrés Rojas, MD, ${ }^{5}$ Alejandra Álvarez, ${ }^{6}$ \\ Luis Miguel Ruiz, ${ }^{6}$ David Restrepo, ${ }^{7}$ Víctor Calvo. ${ }^{8}$
}

1 Cirugía Gastrointestinal y Endoscopia. Jefe de Postgrado Cirugía General U.P.B, Grupo Gastrohepatología Universidad de Antioquia, Instituto de Cancerología Clínica las Américas. MedellínColombia

2 Radiólogo. Internista. Gastroenterólogo. Profesor Universidad de Texas, Campo Regional San Antonio. Director Gastroenterología VA Texas Valley Coastal Bend. Harlingen, Texas

${ }^{3}$ Cirujano General, Clínica Bolivariana. MedellínColombia

${ }^{4}$ Cirujano General, Hospital Pablo Tobón Uribe. Medellín-Colombia

${ }^{5}$ Cirujano General, Instituto de Cancerología-Clínica las Américas. Medellín-Colombia

${ }^{6}$ Estudiantes de Pregrado, Facultad de Medicina U.P.B. Medellín-Colombia

Estudiante de Pregrado, Facultad de Medicina CES. Medellín-Colombia

${ }_{8}$ Estadístico. Medellín-Colombia

\begin{abstract}
Resumen
Introducción: el esófago de Barrett (EB) con displasia tiene un reconocido potencial maligno. Ni la supresión ácida, ni la cirugía antirreflujo producen una regresión consistente o completa del epitelio metaplásico 0 displásico. La termoablación endoscópica a través de la coagulación con argón plasma (APC) ofrece la posibilidad de su erradicación (ablación), pero los posibles factores que pueden influir en el desenlace de la terapia aún no han sido evaluados consistentemente.

Objetivo: evaluar la eficacia de la APC en la erradicación del EB con displasia y los factores que influencian su desenlace inmediato y tras por lo menos un año de seguimiento.

Métodos: A 33 pacientes con EB y displasia (19 hombres-58\%), con una media de edad de 56,9 96,35 años (rango 45 a 69 años) con una longitud media del EB de 4,1 cm (rango de 2-8 cm), se les practicó terapia con APC a intervalos de cada 4-6 semanas, hasta lograr la erradicación del EB, mientras estaban recibiendo terapia con doble dosis de inhibidores de bomba de protones (IBP). Los pacientes se controlaron por endoscopia a los 3, 6 y 12 meses y en adelante cada año, con biopsias de cuatro cuadrantes en las áreas tratadas.

Resultados: la totalidad de los pacientes presentó ablación del EB y la displasia después de una media de 2 sesiones de APC (1 a 4 sesiones). La recurrencia del EB y la displasia se asocian con una mayor longitud inicial del EB circular o de su margen más proximal, a pesar de un mayor número de sesiones de APC (cifras no significativas). A 7 pacientes se les documentó la recaída del Barrett $(21,2 \%)$ y en $3(9,1 \%)$ se presentó nuevamente displasia (dos de bajo grado y una de alto grado) La complicación más frecuente fue el dolor retroesternal presente en 6 pacientes (18,2\%), en 4 hubo estenosis que ameritó dilatación endoscópica (12,1\%) y un paciente $(3 \%)$ presentó sangrado durante el procedimiento que requirió escleroterapia. El seguimiento promedio fue de $43,7 \pm 19$ meses y no se presentó carcinoma de esófago ni hubo ninguna muerte relacionada con la enfermedad o la terapia.

Conclusiones: el tratamiento con APC es un procedimiento seguro y efectivo como terapia ablativa en segmentos cortos del EB con displasia. La recurrencia del EB y la displasia es común luego de un año posterior a la terapia, especialmente en aquellos con segmentos largos de EB.
\end{abstract}

Palabras clave

Esófago de Barrett, reflujo gastroesofágico, argón plasma, displasia en esófago.

\section{INTRODUCCIÓN}

El epitelio o esófago de Barrett (EB) es reconocido como una condición premaligna secundaria al reflujo gastroesofágico crónico (1). Estudios recientes prospectivos de vigilancia endoscópica estiman que la incidencia de un adenocarcinoma esofágico asociada al EB ha disminuido y es de aproximadamente de 1:300 pacientes año $(2,3)$; $\sin$ embargo, la incidencia del adenocarcinoma de esófago se ha incrementado hasta 6 veces en los últimos 30 años (4). Factores de alto riesgo para adenocarcinoma de esófago incluyen un incremento en la longitud del EB (5), el género 


\begin{abstract}
Introduction: Barrett's esophagus (BE) with dysplasia has a recognized potential for malignancy. Neither acid suppression nor anti-reflux surgery produce consistent or complete regression of metaplasia or dysplasia in the epithelium. Endoscopic argon plasma ablation (APC) offers the possibility of eradication, but factors that may influence the outcome of therapy have not been consistently evaluated.

Objective: The objective of this study was to evaluate the efficacy of APC for eradication of BE with dysplasia and to evaluate the factors that influence the immediate outcome and results after one year of follow up.

Methods: The study population included thirty-three patients suffering from BE with dysplasia: 19 men (58\%), mean age of $56.9 \pm 6.35$ years (range 45-69 years) and an average length of EB $4.1 \mathrm{~cm}$ (range 2 to 8 $\mathrm{cm}$.). All patients had undergone APC at intervals of 4 to 6 weeks to eradicate Barrett's esophagus. Patients also received double doses of proton pump inhibitors (PPIs). Patients were endoscopically monitored at 3, 6 and 12 months and thereafter every year with four-quadrant biopsies of treated areas.

Results: Ablation of BE and dysplasia was achieved in all patients after a median of 2 sessions of APC (1-4 sessions). Recurrence of $B E$ and dysplasia is associated with longer initial lengths affected by $B E$ and larger circumferences of its circular or proximal margin, despite treatment with additional APC sessions (no significant differences). Documented recurrences of Barrett's esophagus occurred in seven patients (21.2\%), documented recurrences of dysplasia occurred in three patients (9.1\%). Two of these were low grade and one was high grade. The most frequent complication was chest pain which was experienced by six patients $(18.2 \%)$, four had stenoses that required endoscopic dilatation (12.1\%), and one patient's (3\%) bleeding required sclerotherapy during the procedure. The average follow-up time was $43.7 \pm 19$ months. No esophageal carcinoma developed nor were there any deaths related to the disease or therapy.

Conclusions: Treatment with APC is safe and effective ablative therapy for short segments of BE with dysplasia. Post treatment recurrences of $\mathrm{BE}$ and dysplasia are common especially among patients with long segments of $B E$.
\end{abstract}

\title{
Keywords
}

Barrett's esophagus, gastroesophageal reflux, argon plasma, dysplasia in the esophagus.

masculino (6), la presencia de displasia (7) o hernia hiatal (8), el consumo de alcohol (9) o cigarrillo (10), la obesidad (11) y posiblemente el reflujo duodenogastro-esofágico (12). Por su parte, hay un una relación protectora contra el EB por el consumo de vegetales, folatos y antioxidantes (13).

El manejo actual del EB incluye la vigilancia endoscópica (3), la cual permanece como una medida discutible debido tanto a la morbilidad y mortalidad asociadas con la esofaguectomía para el tratamiento de las displasias de alto grado y los adenocarcinomas tempranos como a la carencia de una evidencia concluyente sobre su costo-efectividad (14). El objetivo de la terapia médica es abolir el reflujo gastroesofágico usando agentes potentes que reduzcan la secreción ácida, tales como los inhibidores de bomba de protones (IBP) (15). También se recomienda la cirugía para el control del reflujo, particularmente para aquellos pacientes con respuestas inadecuadas o intolerantes a la terapia farmacológica (16). Desafortunadamente, ninguna de estas aproximaciones ha mostrado inducir de forma consistente o completa la regresión del $\mathrm{EB}(17,18)$.

Lo anterior ha conducido a explorar nuevas técnicas endoscópicas termoablativas en el contexto de una supresión de la secreción ácida gástrica (19). La coagulación con argón plasma (APC) es un ejemplo de una de estas técnicas, con ella se administra una corriente de alta frecuencia a los tejidos por un flujo de gas argón ionizado (plasma). Esta técnica implica que no es necesario el contacto del catéter con el tejido. El espesor del EB es de $0,6 \mathrm{~mm}$, el cual está dentro de los límites de la terapia por $\operatorname{APC}(20,21)$. Esta terapia con APC ha mostrado algunas ventajas sobre otras intervenciones como el láser o la terapia fotodinámica $(22,23)$ al ser menos costosa, más portátil, y más segura. La coagulación con APC es una técnica ampliamente disponible de bajo costo operacional, fácil de usar en manos de endoscopistas expertos, efectiva y segura, que permite tratar grandes superficies. La tasa de eliminación del epitelio metaplásico es muy promisoria, pero lo pequeño de las series y el corto periodo de seguimiento obligan a calificar con cautela estos resultados $(24,25)$.

El objetivo final de esta terapia es erradicar el tejido con la metaplasia intestinal y reducir el potencial maligno que está relacionado con la presencia del EB. Un limitado número de estudios ha reportado la eficacia de la terapia con APC para el manejo ablativo del EB con displasia o cáncer in situ (2630); y aunque algunos estudios sugieren su aplicación en el EB no displásico (31-37), no está clara su utilidad en muchos otros (22, 38-45); incluso, se ha implementado su uso combinándolo con la cirugía (46-51), pero el seguimiento de estos pacientes es corto y la estabilidad a largo plazo resultante de ese epitelio neoescamoso es incierta (52). 
El objetivo del presente estudio es evaluar la eficacia del APC como terapia ablativa del EB con displasia de alto o bajo grado realizada en varias instituciones de tercer nivel de la ciudad de Medellín, e investigar los factores que influencian el desenlace de la terapia y la estabilidad del epitelio neoescamoso después de por lo menos un año de seguimiento.

\section{PACIENTES Y MÉTODOS}

\section{Tipo de estudio}

Es un estudio observacional descriptivo (serie de casos), en el cual se realizó un seguimiento de los pacientes con diagnóstico de EB con displasia de alto o bajo grado, tratados mediante la ablación endoscópica con APC en dos servicios de endoscopia de tercer nivel de la ciudad de Medellín, en el periodo comprendido entre enero de 2006 y diciembre de 2011.

\section{Diagnóstico de esófago de Barrett}

La totalidad de los pacientes tenía epitelio columnar en el esófago (metaplasia intestinal especializada en la evaluación histológica) por encima de la unión gastroesofágica, la cual fue definida endoscópicamente como aquel tejido que se extendía más de un centímetro por encima de los pliegues más proximales del estómago. La medición del EB se hizo con los criterios de la clasificación de Praga y se midió la distancia del EB circunferencial (BC) así como el margen más proximal del EB (BM). Inicialmente se realizaba un lavado con un mucolítico (N-acetil-cisteina) para lograr una mejor fijación de la tinción con azul de metileno. Con el advenimiento de la cromoendoscopia digital (NBI o FICE) esta tinción fue suprimida. Se realizaron biopsias de los cuatro cuadrantes, escalonadas a intervalos de 1 a $2 \mathrm{~cm}$ para confirmación histológica de EB previo a la ablación, descartar o confirmar la presencia concomitante de displasia de bajo o alto grado; ante la presencia de nodulaciones (posible displasia con lesión levantada) siempre la biopsia se tomó de ellas y durante el seguimiento al completar el estudio para documentar la reepitelialización del epitelio escamoso y para buscar la evidencia de glándulas de EB "sepultado". Todas las muestras fueron evaluadas por mínimo 2 patólogos con especial interés y entrenamiento en la histopatología gastrointestinal (JCP). A ninguno de los pacientes se le hizo estudio con ultrasonido endoscópico.

\section{Criterios de inclusión}

Pacientes con diagnóstico clínico, endoscópico e histopatológico de $\mathrm{EB}$ con displasia de cualquier grado y con edades entre 18 y 70 años.

\section{Criterios de exclusión}

Se excluyeron el estudio los pacientes que se rehusaron a participar, aquellos a los cuales es imposible seguirlos en el tiempo, pacientes con enfermedad terminal, cirugías previas en esófago o con adenocarcinoma invasor de esófago.

\section{Muestra}

El muestreo es no probabilístico de casos continuos; es decir, ingresaron al estudio en forma consecutiva todos los pacientes que cumplían con el diagnóstico de EB con displasia, manejados con ablación por coagulación endoscópica con APC en los servicios endoscópicos descritos.

\section{Técnica de recolección}

La información se recolectó de forma retrospectiva y prospectiva a partir de los registros detallados de las historias clínicas; el reporte de las endoscopias y el resultado de la histopatología de los pacientes que cumplían los criterios de inclusión, se hizo a través de una herramienta diseñada previamente por los investigadores en la cual se consignaron todos los datos.

\section{Medicamentos}

A todos los pacientes se les suministró un régimen de IBP, usualmente omeprazol o lansoprazol $20 \mathrm{mg}$ o $30 \mathrm{mg}$ dos veces al día para alcanzar una adecuada supresión del ácido. En pacientes con reflujo ácido persistente, a pesar de los esquemas de tratamiento anteriores, la dosis del IBP se incrementó.

\section{Coagulación con argón plasma}

La endoscopia se realizó en forma ambulatoria usando sedación con midazolam IV ( 3 a 5 mg), meperidina IV (20$50 \mathrm{mg}$ ) y butilbromuro de hioscina 10 a $20 \mathrm{mg}$ IV para inhibir la peristalsis esofágica. Se monitorizó la pulso-oximetría y la presión sanguínea durante todo el procedimiento. La terapia se realizó usando un sistema Erbe APC 200 (Erbe Medical UK Ltd, Leeds, UK) y una sonda gastrointestinal flexible Erbe. La terapia con APC fue liberada a una intensidad ajustada de 50 watt a un flujo de $11 / \mathrm{min}$, con intervalos mensuales con el objetivo de tratar hasta dos tercios de la circunferencia esofágica por sesión, hasta el que el aclaramiento macroscópico fuese alcanzado. Para los segmentos más cortos y para las islas de $\mathrm{EB}$, se utilizó una aplicación puntual mientras que para las áreas más grandes se empleó una terapia con la sonda en dirección cráneo-caudal para lograr un coágulo blanco por la lesión térmica (figura 1). 

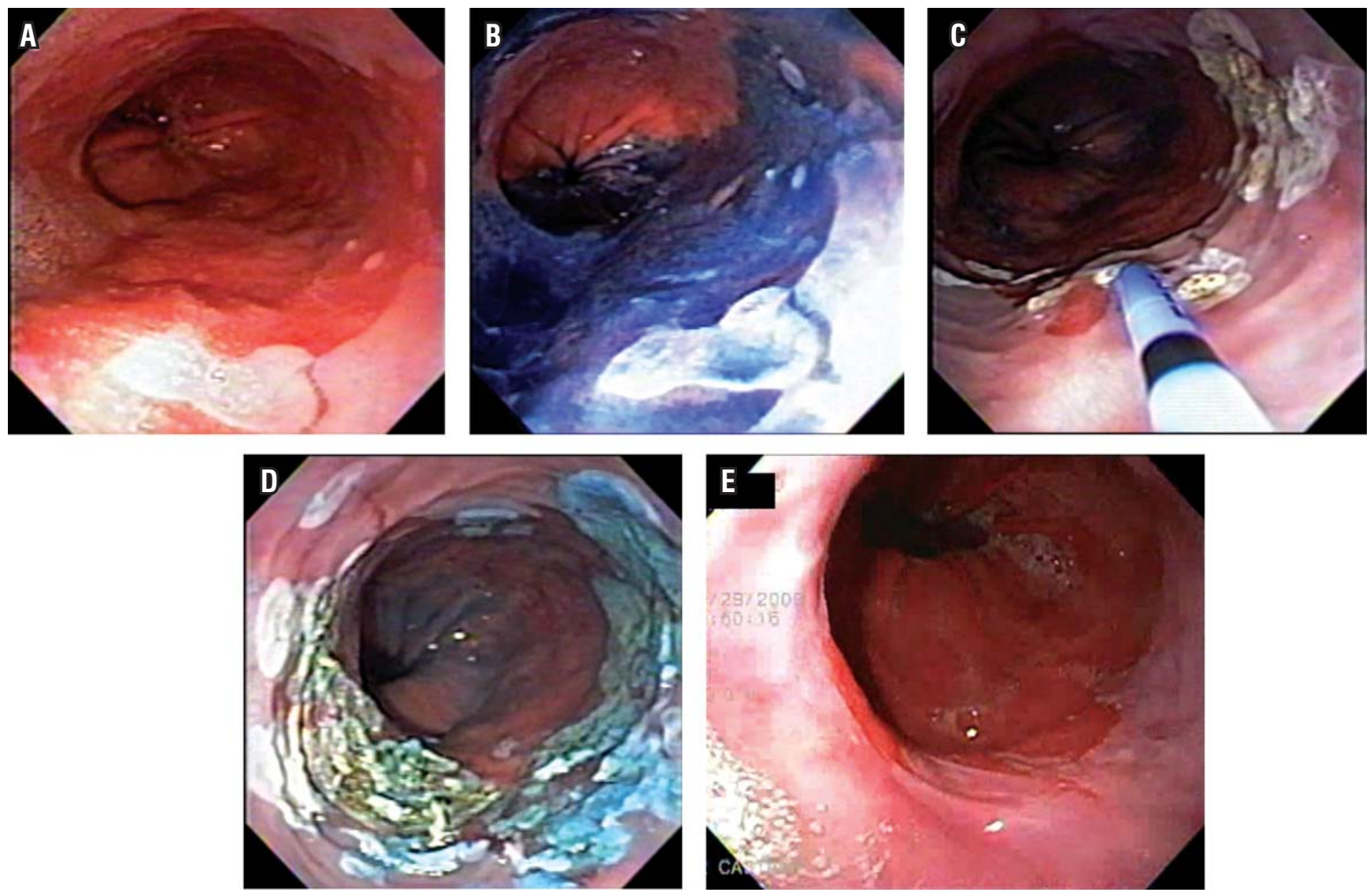

Figura 1. Aspectos endoscópicos en la terapia con APC y EB con DBG. A. Esófago de Barrett largo con displasia de bajo grado, C3M4 en la clasificación de Praga. B. Tinción con azul de metileno para destacar los márgenes y sitios que sugieran displasia. C. Inicio de la terapia con argón plasma en el margen oral del esófago de Barrett. D. Se completa la terapia con argón plasma de $2 / 3$ de la circunferencia del esófago de Barrett. E. Endoscopia de seguimiento sin EB al año tras la terapia de erradicación con argón plasma.

Se registró cualquier síntoma relacionado con el procedimiento por parte del paciente y adicionalmente se contactó telefónicamente 24 horas postintervención para evaluar la presencia de complicaciones. Se definió el fracaso terapéutico como la persistencia de áreas originales de Barrett >10\% a pesar del tratamiento ablativo por lo menos luego de 2 ocasiones. Por otro lado, las áreas de terapia ablativa después de 2 sesiones, en las que no hubo reepitelialización escamosa, también se consideró un fracaso terapéutico. Los pacientes en quienes se documentó algún efecto benéfico de la terapia continuaron en el estudio hasta un máximo de 4 sesiones.

\section{Seguimiento a un año}

Los pacientes fueron educados y estimulados para continuar la misma dosis de IBP que estaba siendo usada durante el estudio y se les practicó una endoscopia de revisión a los 3, 6 y 12 meses de haber sido completado el tratamiento, durante el cual la extensión del EB, si lo hubiese, fue documentado y adicionalmente se tomaron biopsias de acuerdo con el protocolo de biopsia de cuatro cuadrantes cada dos centímetros (protocolo Seattle).

\section{Análisis estadístico}

En el análisis descriptivo se utilizaron distribuciones absolutas y relativas e indicadores de resumen (medidas de tendencia central, de posición, de dispersión). Se utilizó la prueba Chi cuadrado de independencia, la prueba Exacta de Fisher o la Razón de verosimilitudes cuando fuere necesario. Se estableció el criterio de normalidad de los datos mediante la prueba de Kolmogorov-Smirnov y con base en esta se aplicó la prueba t-Student para la diferencia de medias; un valor $\mathrm{p}$ inferior de 0,05 se consideró estadísticamente significativo. Para el análisis de supervivencia se aplicó el método de Kaplan-Meier y para la comparación de curvas se utilizó Log Rank (Mantel-Cox) o Breslow (Wilcoxon Generalizado). La sistematización, el procesamiento y análisis de los datos se realizó mediante el programa estadístico SPSS versión 17. 


\section{Consentimiento informado y aspectos éticos}

Todo paciente incluido en el estudio firmó consentimiento informado antes de recibir la terapia endoscópica con APC. El estudio fue aprobado por el comité de ética de la Universidad de Antioquia que evalúa los trabajos realizados por los residentes para optar al título de especialista.

\section{RESULTADOS}

En el periodo comprendido entre abril de 2004 y septiembre de 2009 entraron al estudio un total de 33 pacientes con EB con displasia de alto (10 pacientes-30\%) o bajo grado (23 pacientes-70\%); recibieron tratamiento endoscópico ablativo con APC, todos los pacientes siguieron recibiéndolo después del procedimiento IBP a dosis altas.

En los 33 pacientes con EB se obtuvo una respuesta completa con regeneración del epitelio escamoso al final del tratamiento dada por la falta de evidencia endoscópica de segmentos de epitelio columnar y con la confirmación histológica de la presencia del epitelio escamoso. El seguimiento promedio fue de $43,7 \pm 19$ meses; el número de sesiones requeridas fue de 1 sesión en 3 pacientes (9,1\%), 2 en 19 pacientes (57,6\%), 3 en 11 pacientes (33,3\%).

La recurrencia se presentó en $30,3 \%$ de los pacientes, nuevo Barret en 7 (21,2\%) y nueva displasia en $3(9,1 \%)$; no se encontró en las biopsias progresión a malignidad durante el tiempo de seguimiento.

Las complicaciones se documentaron en 33,3\% de los pacientes, discriminadas por dolor en 6 pacientes (18,2\%), el cual se autolimitó o cedió al manejo analgésico; estenosis en $4(12,1 \%)$ y sangrado durante el procedimiento en 1 (3\%), el cual ameritó intervención endoscópica con escleroterapia. Durante el seguimiento no se reportó mortalidad asociada al tratamiento y/o a la enfermedad. Las características generales del grupo se recogen en la tabla 1.

El resultado final tras la terapia con APC muestra que en 7 pacientes reapareció el Barrett y en 3 pacientes la displasia recurrió. No se presentó cáncer en ninguno de los pacientes tratados (figuras 2 y 3 ).

En los pacientes con displasia de bajo grado, 3 pacientes $(13,0 \%)$ presentaron nuevo EB al final del tratamiento y de aquellos con displasia de alto grado, $4(40,0 \%)$ presentaron nuevo EB.

De los pacientes con DBG, 1 (4,3\%) presentó nueva displasia de bajo grado al final del tratamiento y de los pacientes con DAG, $2(20,0 \%)$ presentaron nueva displasia de bajo y alto grado.

El promedio de edad de los pacientes en el presente estudio fue de $56,9 \pm 6,35$ años. De igual manera, $50 \%$ de los pacientes presentó un tiempo de RGE de 8 años o menos. Es importante destacar el promedio de tiempo de
Tabla 1. Distribución absoluta y porcentual de variables sociodemográficas y antecedentes clínicos.

\begin{tabular}{|c|c|c|c|}
\hline & Categoría & $\mathrm{n}$ & $\%$ \\
\hline \multirow[t]{2}{*}{ Sexo } & Hombre & 19 & 57,6 \\
\hline & Mujer & 14 & 42,4 \\
\hline \multirow[t]{2}{*}{ Grupos de edad } & > 50 años & 26 & 78,8 \\
\hline & $\leq 50$ años & 7 & 21,2 \\
\hline \multirow[t]{2}{*}{ Cirugía Anti-RGE } & $\mathrm{Si}$ & 8 & 24,2 \\
\hline & No & 25 & 75,8 \\
\hline \multirow[t]{2}{*}{ Hernia hiatal } & $\mathrm{Si}$ & 21 & 63,6 \\
\hline & No & 12 & 36,4 \\
\hline \multirow[t]{2}{*}{ Nódulos } & $\mathrm{Si}$ & 9 & 27,3 \\
\hline & No & 24 & 72,7 \\
\hline \multirow[t]{3}{*}{ Número de sesiones } & Una sesión & 3 & 9,1 \\
\hline & Dos sesiones & 19 & 57,6 \\
\hline & Tres sesiones & 11 & 33,3 \\
\hline \multirow[t]{2}{*}{ Displasia } & Bajo grado & 23 & 69,7 \\
\hline & Alto grado & 10 & 30,3 \\
\hline \multirow{5}{*}{$\begin{array}{l}\text { Barrett circunferencial } \\
\text { (BC) }\end{array}$} & 1 & 7 & 21,2 \\
\hline & 2 & 12 & 36,4 \\
\hline & 3 & 9 & 27,3 \\
\hline & 4 & 4 & 12,1 \\
\hline & 5 & 1 & 3,0 \\
\hline \multirow{7}{*}{$\begin{array}{l}\text { Barrett y su margen } \\
\text { (BM) }\end{array}$} & 1 & 1 & 3,0 \\
\hline & 2 & 8 & 24,2 \\
\hline & 3 & 12 & 36,4 \\
\hline & 4 & 7 & 21,2 \\
\hline & 5 & 2 & 6,1 \\
\hline & 6 & 2 & 6,1 \\
\hline & 7 & 1 & 3,0 \\
\hline \multirow[t]{4}{*}{ Complicaciones } & No & 22 & 66,7 \\
\hline & Dolor & 6 & 18,2 \\
\hline & Estenosis & 4 & 12,1 \\
\hline & Sangrado & 1 & 3,0 \\
\hline \multirow[t]{2}{*}{ Cirugía } & $\mathrm{Si}$ & 8 & 24,2 \\
\hline & No & 25 & 75,8 \\
\hline
\end{tabular}

tratamiento desde el inicio de sesión hasta la terminación que fue de $141 \pm 73,5$ días, y el promedio de seguimiento de dichos pacientes hasta el último control fue de $43,7 \pm 19$ meses (tabla 2).

En las tablas 3 y 4 se recogen la distribución absoluta y porcentual de los antecedentes y las circunstancia clínicas según la presencia de nuevo esófago de Barrett o de displasia.

El promedio de tiempo con RGE en los pacientes que presentaron nuevo Barrett fue de $9,3 \pm 2,8$ y el de los que no lo presentaron fue de $7,2 \pm 3,5$, no se hallaron diferencias 


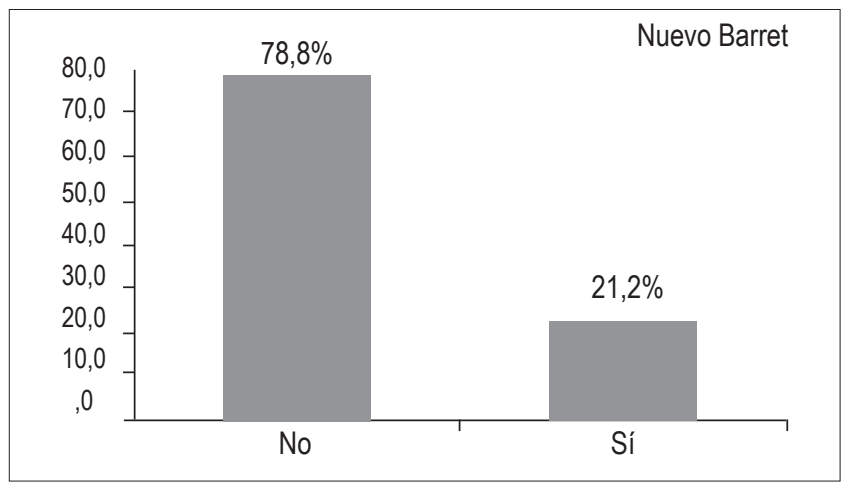

Figura 2. Distribución porcentual de nuevo Barrett, después de tratamiento.

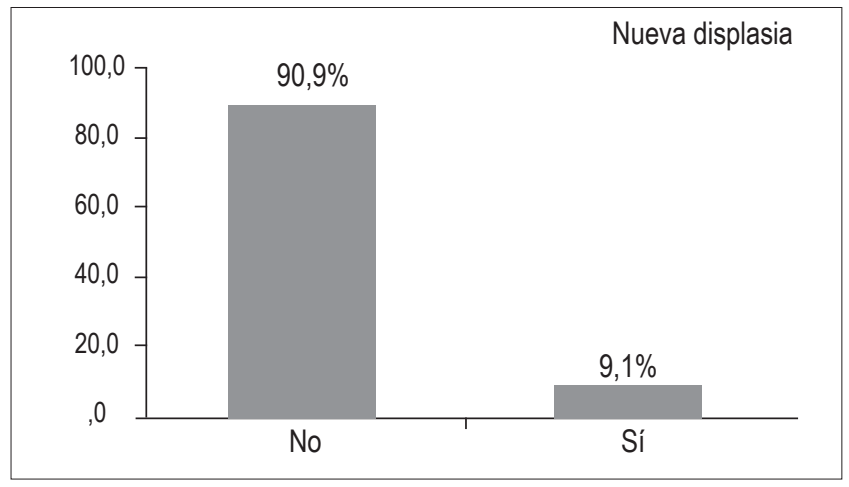

Figura 3. Distribución porcentual de nueva displasia, después de tratamiento.

Tabla 2. Distribución de variables persona, circunstancia clínica y de tiempo $(n=33)$.

\begin{tabular}{lccccc}
\hline & Media aritmética & Desviación estándar & Mediana & Mínimo & Máximo \\
\hline Edad & 56,9 & 6,35 & 57,0 & 45 & 67 \\
Años con RGE & 7,6 & 3,43 & 8,0 & 2 & 16 \\
Paq/año (n=15) & 7,5 & 3,12 & 9,0 & 3 & 12 \\
IMC & 28,7 & 2,79 & 28,0 & 24 & 33 \\
Días tratamiento $^{1}$ & 141 & 73,5 & 123 & 0 & 303 \\
Meses seguimiento $^{2}$ & 43,7 & 19,16 & 44,0 & 15 & 78 \\
\hline
\end{tabular}

${ }^{1}$ Inicio sesión hasta terminación sesión

${ }^{2}$ Inicio sesión hasta último control.

Tabla 3. Distribución absoluta y porcentual de antecedentes, circunstancia clínicas según la presencia de nuevo esófago de Barrett $(n=33)$.

\begin{tabular}{llcccc}
\hline & & \multicolumn{2}{c}{ Nuevo Barrett } & Chi $^{2}$ & p \\
\cline { 3 - 4 } & & SI (\%) & NO (\%) & & \\
\hline \multirow{2}{*}{ Sexo } & Hombre & $5(26,3)$ & $14(73,7)$ & 0,698 & 0,670 \\
& Mujer & $2(14,3)$ & $12(85,7)$ & & \\
Anti-RGE & Si & $2(13,3)$ & $13(86,7)$ & 1,021 & 0,413 \\
& No & $5(27,8)$ & $13(72,2)$ & & \\
HH & Si & $2(25,0)$ & $6(75,0)$ & 0,091 & 0,763 \\
& No & $5(20,0)$ & $20(80,0)$ & & \\
Nódulos & Si & $3(14,3)$ & $18(85,7)$ & 1,658 & 0,377 \\
& No & $4(33,3)$ & $8(66,7)$ & & \\
Displasia & Si & $3(33,3)$ & $6(66,7)$ & 1,088 & 0,358 \\
& No & $4(16,7)$ & $20(83,3)$ & & \\
Complicación & Bajo grado & $3(13,0)$ & $20(87,0)$ & 3,030 & 0,161 \\
& Alto grado & $4(40,0)$ & $6(60,0)$ & & \\
& No & $5(22,7)$ & $17(77,3)$ & 3,898 & 0,173 \\
& Dolor & $0(0,0)$ & $6(100,0)$ & & \\
& Estenosis & $2(50,0)$ & $2(50,0)$ & & \\
Cirugía & Sangrado & $0(0,0)$ & $1(100,0)$ & & \\
& Si & $1(12,5)$ & $7(87,5)$ & 0,480 & 0,652 \\
& No & $6(24,0)$ & $19(76,0)$ & & \\
& Si & $1(33,3)$ & $2(66,7)$ & 0,290 & 0,523 \\
& Ni & $6(20,0)$ & $24(80,0)$ & & \\
& No & & & &
\end{tabular}

Tabla 4. Distribución absoluta y porcentual de antecedentes, circunstancia clínicas según la presencia de nueva displasia $(\mathrm{n}=33)$.

\begin{tabular}{|c|c|c|c|c|c|}
\hline & & \multicolumn{2}{|c|}{ Nueva displasia } & \multirow[t]{2}{*}{$\mathrm{Chi}^{2}$} & \multirow[t]{2}{*}{$P$} \\
\hline & & SI n (\%) & NO n (\%) & & \\
\hline \multirow[t]{2}{*}{ Sexo } & Hombre & $2(10,5)$ & $17(89,5)$ & 0,112 & 1,000 \\
\hline & Mujer & $1(7,1)$ & $13(92,9)$ & & \\
\hline \multirow[t]{2}{*}{ Fuma } & $\mathrm{Si}$ & $2(13,3)$ & $13(86,7)$ & 0,599 & 0,579 \\
\hline & No & $1(5,6)$ & $17(94,4)$ & & \\
\hline \multirow[t]{2}{*}{ Anti-RGE } & $\mathrm{Si}$ & $0(0,0)$ & $8(100,0)$ & 1,056 & 0,560 \\
\hline & No & $3(12,0)$ & $22(88,0)$ & & \\
\hline \multirow[t]{2}{*}{$\mathrm{HH}$} & $\mathrm{Si}$ & $2(9,5)$ & $19(90,5)$ & 0,013 & 1,000 \\
\hline & No & $1(8,3)$ & $11(91,7)$ & & \\
\hline \multirow[t]{2}{*}{ Nódulos } & $\mathrm{Si}$ & $3(33,3)$ & $6(66,7)$ & 8,800 & 0,015 \\
\hline & No & $0(0,0)$ & $24(100,0)$ & & \\
\hline \multirow[t]{2}{*}{ Displasia } & Bajo grado & $1(4,3)$ & $22(95,7)$ & 2,066 & 0,212 \\
\hline & Alto grado & $2(20,0)$ & $8(80,0)$ & & \\
\hline \multirow[t]{4}{*}{ Complicación } & No & $2(9,1)$ & $20(90,9)$ & 1,295 & $0,730^{*}$ \\
\hline & Dolor & $1(16,7)$ & $5(83,3)$ & & \\
\hline & Estenosis & $0(0,0)$ & $4(100,0)$ & & \\
\hline & Perforación & $0(0,0)$ & $1(100,0)$ & & \\
\hline \multirow[t]{2}{*}{ Cirugía } & $\mathrm{Si}$ & $1(12,5)$ & $7(87,5)$ & 0,149 & 1,000 \\
\hline & No & $2(8,0)$ & $23(92,0)$ & & \\
\hline
\end{tabular}

* Razón de verosimilitudes. 
significativas entre dichas variables $(p>0,05)$; este mismo hecho ocurrió con las variables edad e IMC, días de tratamiento y meses de seguimiento (tabla 5).

Tabla 5. Distribución variables cuantitativas según nuevo Barrett $(n=33)$.

\begin{tabular}{|c|c|c|c|c|}
\hline & \multicolumn{2}{|c|}{ Nuevo Barrett } & \multirow[t]{2}{*}{$\mathrm{t}$-Student } & \multirow{2}{*}{$\begin{array}{c}\text { Valor } \\
\mathrm{p}\end{array}$} \\
\hline & SI & NO & & \\
\hline Edad & $59,4 \pm 3,7$ & $56,3 \pm 6,8$ & 1,175 & 0,249 \\
\hline Años con RGE & $9,3 \pm 2,8$ & $7,2 \pm 3,5$ & 1,460 & 0,154 \\
\hline IMC & $28,9 \pm 1,9$ & $28,6 \pm 3,0$ & 0,169 & 0,867 \\
\hline Días tratamiento* & $143,6 \pm 85,1$ & $140,5 \pm 71,7$ & 0,098 & 0,923 \\
\hline $\begin{array}{l}\text { Meses } \\
\text { seguimiento** }\end{array}$ & $34,9 \pm 17,5$ & $46,1 \pm 19,2$ & 1,397 & 0,172 \\
\hline
\end{tabular}

*Inicio sesión hasta terminación sesión

** Inicio sesión hasta último control.

Los pacientes que presentaron recaída de la displasia y del EB presentaron por lo menos un centímetro más de longitud del EB, tanto en su compromiso circunferencial (BC de la clasificación de Praga) como en su margen proximal (BM de la clasificación de Praga).

En las figuras 4 y 5 se grafican las curvas de supervivencia para el grupo en general y discriminados por sexo.

\section{DISCUSIÓN}

La presencia del EB es la complicación más seria observada en el RGE crónico, situación ya evaluada en nuestro medio por el doctor Sierra (2), que como condición premaligna aumenta el riesgo de forma significativa del adenocarcinoma de esófago (7). En las últimas dos décadas se han realizado grandes esfuerzos en el desarrollo de tratamientos

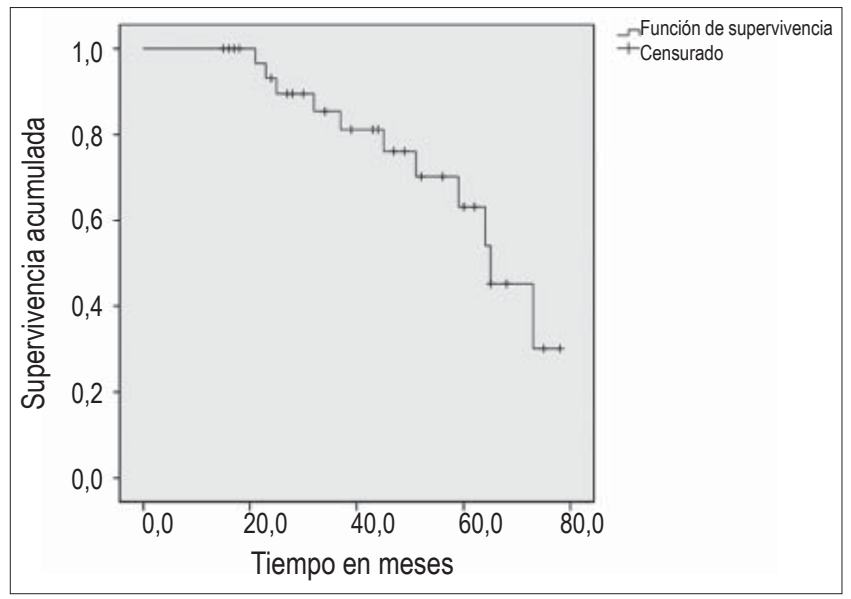

Figura 4. Análisis de supervivencia general. endoscópicos, en especial en el campo de la terapia ablativa, considerada en la actualidad una opción terapéutica válida por las diferentes asociaciones médicas que definen el manejo del EB, debido a que el tratamiento estándar quirúrgico (esofaguectomía) de los pacientes con DAG tiene una tasa de morbilidad reportada hasta de $47 \%$ y de mortalidad en las diferentes series de $1,2 \%$ a $4 \%$, comparada con aquella de los diferentes tratamientos endoscópicos la cual es de $0,04 \%$ (33).

La coagulación por APC induce la regeneración escamosa en el EB, la cual no presenta diferencias con el epitelio de la mucosa del esófago sano, soportado por varios hallazgos moleculares (53). La terapia ablativa por este método usualmente es bien tolerada por la mayoría de los pacientes con escasos efectos secundarios autolimitados y una tasa de complicaciones baja. Sin embargo, no existe consenso en la intensidad de la terapia con APC y se han comparado voltajes de 50 y $70 \mathrm{~W}$ sin demostrase una mayor tasa de penetración en la pared, ni diferencias en el dolor posprocedimiento (54).

En 1997 se publicaron los primeros estudios de la terapia ablativa del EB sin displasia con APC por Dumoulin (55), seguidas por los estudios de Mork (56) y Byrne (24) con tasas de erradicación de $100 \%$ del EB y del epitelio displásico; la primera terapia del EB con displasia fue reportada por Maass (57). Las diferentes series publicadas a la fecha reportan una erradicación completa del EB sin displasia que oscila entre $36 \%$ y $100 \%$ con seguimientos a corto plazo. Madish (33) muestra en 70 pacientes con EB sin displasia una erradicación de $98 \%$, con una tasa de recurrencia de $12 \%$ y una media de seguimiento de 51 meses. Ferraris (52) documenta una respuesta completa de $97 \%$, con una recidiva de $6,1 \%$ anual asociado a la fundoplicatura laparoscópica como un factor protector. En contraste otro

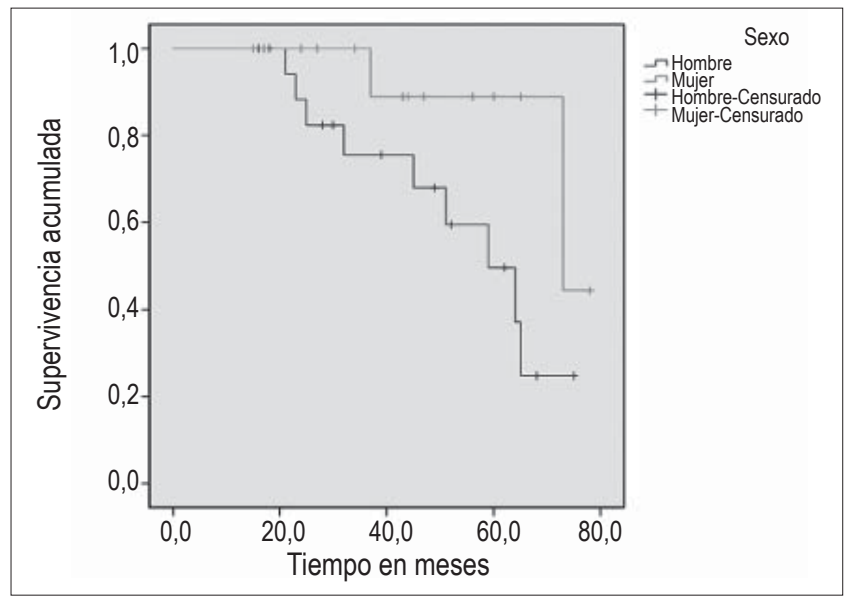

Figura 5. Análisis de supervivencia según sexo. Log Rank (MantelCox=3.222; $\mathrm{p}=0,073$ ) 
estudio publicado por Mork (41) reporta una cura de $84 \%$ con una recurrencia de $66 \%$ en 25 pacientes con un tiempo de seguimiento similar. En nuestro estudio, se encuentra una erradicación del EB displásico de 100\% y se observa una recidiva de 30,3\% (20,2\% nuevo Barret y 9,1\% nueva displasia) con un promedio de seguimiento de 43 meses.

Manner (58) utilizó APC de alta energía $90 \mathrm{~W}$ para tratar 51 pacientes con EB sin displasia, 9 pacientes (18\%) presentaron complicaciones menores incluyendo odinofagia, dolor retroesternal, fiebre y odinofagia; en el mismo estudio se documentaron complicaciones mayores incluyendo hemorragia, estenosis y perforación en 5 pacientes (10\%). En nuestra experiencia se observó una tasa global de complicaciones de $33,3 \%$ : dolor $(18,2 \%)$, estenosis $(12,1 \%)$ y sangrado (3\%), muy similar a la evidenciada en diferentes publicaciones $(24,56)$.

Bright (38) comparó la terapia endoscópica con APC versus la vigilancia endoscópica en pacientes con $\mathrm{EB}$ sin displasia bajo terapia antisecretora ácida. Se evaluó la recurrencia del EB a los 12 meses de seguimiento. Se logró en 95\% la ablación de la metaplasia ( 25 de los 26 pacientes tratados). A los 12 meses, 14 de 23 pacientes tratados con APC tenían al menos 95\% de regresión, y nueve de los 23 tuvo una regresión completa del EB. Ningún paciente sometido a seguimiento endoscópico tuvo más de $5 \%$ de regresión. La longitud del esófago de Barrett se acorta significativamente después de APC (media 3,0 frente a 0,5 $\mathrm{cm}$ ). Un retroceso significativo del esófago de Barrett sigue a la ablación con APC, aunque la regresión completa se logró en menos de la mitad del grupo. Este mismo autor comparó la terapia con APC y la cirugía antirreflujo; tras 68 meses de seguimiento se demuestra que en la mayoría de pacientes tratados con APC el epitelio neoescamoso se mantiene más estable y la aparición de la displasia solo se dio en los pacientes quirúrgicos (59).

Sin embargo, la ablación en el EB no neoplásico sigue siendo un tópico que genera controversia, especialmente en el impacto que tiene sobre el riesgo de la progresión de la enfermedad hacia el adenocarcinoma, incluso existen reportes de adenocarcinoma por debajo del epitelio escamoso restaurado posterior al tratamiento (60-62), y hasta carcinoma escamoso en el área tratada (63).

Los pacientes con EB frecuentemente presentan trastornos en la motilidad del esófago, con disminución del aclaramiento esofágico, sumado a una disminución en la sensibilidad y severidad de los síntomas. Se evaluaron con manometría del esófago por Smythe (64) 20 pacientes con EB sometidos a terapia con APC sin demostrarse sustantivos cambios en la motilidad del esófago tras la terapia con APC, pero la evaluación de la sensibilidad con diferentes soluciones mostró una significativa menor sensibilidad al ácido y al álcali en el grupo tratado. El estudio de Basu tampoco mostró afectación de la motilidad en los pacientes con EB tratados con APC (65).

En un reciente estudio de costos en que se compara el seguimiento endoscópico con diferentes terapias ablativas en el paciente con EB, EB con DBG y EB con DAG, se demuestra que la ablación endoscópica debe ser la estrategia preferida en el paciente con EB y DAG. La ablación también debe ser de elección en el paciente con DBG o aun sin displasia, pero el costo-efectividad de que no sea necesario el seguimiento endoscópico por una efectiva erradicación del EB (66).

Se ha comparado la terapia ablativa del EB con APC versus la mucosectomía con bandas por Boix (67), sugiriendo un mejor desenlace con la mucosectomía endoscópica sobre la terapia con APC, con un mayor número de sesiones pero con un mayor porcentaje de erradicación del epitelio metaplásico. Estos autores habían sugerido una terapia combinada que se inicia con la resección endoscópica mucosa con bandas seguida por la terapia ablativa con APC en los casos que sean necesarios (islotes de EB remanentes) (68).

La intensidad del voltaje usado en las diferentes series oscila entre 30 y $90 \mathrm{~W}$ y los estudios con los niveles más bajos tiene el mayor índice de "EB sepultado" y solo 68\% de regresión y con una alta tasa de recurrencia (69). Pereira-Lima muestra reepitelialización de $100 \%$ y ausencia de "EB sepultado", con un voltaje de 65-70 W, sin encontrar un número mayor de complicaciones (27). Shulz utiliza el máximo voltaje de $90 \mathrm{~W}$ con ablación completa del epitelio metaplásico en $98,6 \%$ (34). La mayoría de estudios utilizan de 50-60 Wy en una reciente revisión las complicaciones descritas a estos niveles son una muerte por perforación, 2 perforaciones con sobrevida, un enfisema subcutáneo sin evidencia clínica de perforación y 7 estenosis en aproximadamente 500 pacientes evaluados (70). Quejas menores son la odinofagia y disfagia con dolor retroesternal frecuente.

El procedimiento puede hacerse bajo sedación consciente y la anestesia general no se recomienda como sí lo sugiere Tigges (51).

La principal preocupación actual en la terapia ablativa con APC es el epitelio del EB recurrente. La metaplasia subyacente o "profunda" es el potencial neoplásico en el nuevo epitelio escamoso; aunque algunos estudios sugieren que tienen un bajo potencial de transformación maligna, en los especímenes histopatológicos de nuestro estudio se describieron lesiones subepiteliales en el 20,2\%. Los estudios moleculares sugieren que los pacientes con EB, la expresión del oncogén miR-143 todavía está elevada tanto en la mucosa neoescamosa, como en la mucosa escamosa encima del segmento metaplásico, lo que sugiere que esta mucosa puede no ser normal, es decir, que es diferente a la observada en sujetos sin esófago de Barrett. El miR-143 podría promover un patrón de expresión génica del epite- 
lio de Barrett, y esto podría tener un papel en el desarrollo del esófago de Barrett (71). La evaluación del p53 antes y después de la terapia con APC en paciente con EB sin displasia mostró que el p53 era altamente prevalente en el epitelio escamoso contiguo al EB. Después de la ablación, ninguno de los casos con pérdida de la expresión del p53 y ninguno de los casos negativos se tornaron positivos para el p53 (72).

Dos factores influencian el éxito a largo plazo de la coagulación por APC en el EB y son la energía térmica administrada $(50 \mathrm{~W})$ y el uso de altas dosis de IBP, los cuales han marcado una diferencia en la recaída durante el seguimiento en los diferentes artículos publicados, lo que fue tenido en cuenta en el presente estudio. Por otro lado, el presente estudio ratifica lo reportado por Kahaleh (45) y Basu (69) que los dos factores que determinan una pronta recaída en los pacientes con EB tratados con APC son la persistencia del reflujo gastroesofágico y una longitud del Barrett inicial mayor a $3 \mathrm{~cm}$.

La terapia con APC en el EB es un procedimiento con muchas ventajas como que puede hacerse ambulatoriamente, es de corta duración, a diferencia de la terapia fotodinámica no requiere una modificación sustancial de los hábitos de vida, es barato, y el equipo puede tener otros múltiples usos.

\section{CONCLUSIONES}

La eficacia del tratamiento ablativo endoscópico con APC en el EB con y sin displasia es de 100\% (logró la ablación completa de la lesión), con una tasa de recaída de 30,3\% (nuevo EB en $21,2 \%$, nueva displasia 9,1\%), con un seguimiento promedio de 43 meses no hubo progresión de la enfermedad a adenocarcinoma. No se logró determinar que los factores de riesgo asociados a la enfermedad por RGE y el EB, tuvieran un impacto y/o asociación en la recurrencia posterior al tratamiento ablativo con APC. El número de sesiones requerido para la ablación completa con el APC son de 1 a 3 , siendo 2 en la mayoría de pacientes (56,7\%), con mayor número de sesiones en los pacientes con EB más largos (mayor a $3 \mathrm{~cm}$ ) y con una mayor tasa de recurrencia del EB y de la displasia en los EB más largos. La morbilidad atribuida a la coagulación con APC asciende a 33,3\%; no se reportó mortalidad por la enfermedad o secundaria al tratamiento. Es necesario realizar ensayos clínicos controlados y aleatorizados, con un mayor número de pacientes y seguimiento a largo plazo, para determinar la real eficacia y las tasas de recurrencia.

\section{Conflictos de intereses}

Los autores no presentan ningún conflicto de interés.
Este estudio se realizó con el apoyo del Proyecto sostenibilidad de la vicerrectoría de investigación de la Universidad de Antioquia.

\section{REFERENCIAS}

1. Spechler SJ, Goyal RK. The columnar-lined esophagus, intestinal metaplasia, and Norman Barrett. Gastroenterology 1996;110: 614-21.

2. Sierra F. Incidencia de adenocarcinoma en esófago de Barrett, Fundación Santa Fe de Bogotá, 11 años de seguimiento. Rev Col Gastroenterol 2008; 23: 13-25.

3. Grant KS, Demeester SR, Kreger V, et al. Effect of Barrett's esophagus surveillance on esophageal preservation, tumor stage, and survival with esophageal adenocarcinoma. J Thorac Cardiovasc Surg 2013.

4. Solaymani-Dodaran M, Card TR, West J. Cause-Specific Mortality of People With Barrett's Esophagus Compared With the General Population: A Population-Based Cohort Study. Gastroenterology 2013.

5. Pohl H, Wrobel K, Bojarski C, et al. Risk factors in the development of esophageal adenocarcinoma. Am J Gastroenterol 2013; 108: 200-7.

6. Rubenstein $\mathrm{JH}$, Morgenstern $\mathrm{H}$, Appelman $\mathrm{H}$, et al. Prediction of Barrett's Esophagus Among Men. Am J Gastroenterol 2013.

7. Sikkema M, Looman CW, Steyerberg EW, et al. Predictors for neoplastic progression in patients with Barrett's Esophagus: a prospective cohort study. Am J Gastroenterol 2011; 106: 1231-8.

8. Andrici J, Tio M, Cox MR, Eslick GD. Hiatal Hernia and the Risk of Barrett's Esophagus: A Meta-Analysis. Journal of gastroenterology and hepatology 2012.

9. Hardikar S, Onstad L, Blount PL, Odze RD, Reid BJ, Vaughan TL. The role of tobacco, alcohol, and obesity in neoplastic progression to esophageal adenocarcinoma: a prospective study of Barrett's esophagus. PloS one 2013; 8: e52192.

10. Cook MB, Shaheen NJ, Anderson LA, et al. Cigarette smoking increases risk of Barrett's esophagus: an analysis of the Barrett's and Esophageal Adenocarcinoma Consortium. Gastroenterology 2012; 142: 744-53.

11. El-Serag HB, Hashmi A, Garcia J, et al. Visceral abdominal obesity measured by CT scan is associated with an increased risk of Barrett's oesophagus: a case-control study. Gut 2013.

12. Cheng P, Li JS, Gong J, Zhang LF, Chen RZ. Effects of refluxate $\mathrm{pH}$ values on duodenogastroesophageal refluxinduced esophageal adenocarcinoma. World J Gastroenterol 2011; 17: 3060-5.

13. Sun Q Huang Q, Feng AN, et al. Columnar-lined esophagus in Chinese patients with proximal gastric carcinomas. Journal of digestive diseases 2013; 14: 22-8.

14. De Palma GD. Management strategies of Barrett's esophagus. World J Gastroenterol 2012; 18: 6216-25.

15. Kastelein F, Spaander MC, Steyerberg EW, et al. Proton Pump Inhibitors Reduce the Risk of Neoplastic Progression 
in Patients With Barrett's Esophagus. Clin Gastroenterol Hepatol 2012.

16. Marano S, Mattacchione S, Luongo B, Paltrinieri G, Mingarelli V, Tosato F. Barrett's esophagus after laparoscopic Nissen-Rossetti fundoplication: functional evaluation. Minerva chirurgica 2011; 66: 517-25.

17. Bennett C, Green S, Decaestecker J, et al. Surgery versus radical endotherapies for early cancer and high-grade dysplasia in Barrett's oesophagus. Cochrane Database Syst Rev 2012; 11: CD007334.

18. Simonka Z, Paszt A, Abraham S, et al. The effects of laparoscopic Nissen fundoplication on Barrett's esophagus: longterm results. Scand J Gastroenterol 2012; 47: 13-21.

19. Bergman JJ, Corley DA. Barrett's esophagus: who should receive ablation and how can we get the best results? Gastroenterology 2012; 143: 524-6.

20. Ackroyd R, Brown NJ, Stephenson TJ, Stoddard CJ, Reed MW. Ablation treatment for Barrett oesophagus: what depth of tissue destruction is needed? Journal of clinical pathology 1999; 52: 509-12.

21. Watson JP, Bennett MK, Griffin SM, Matthewson K. The tissue effect of argon plasma coagulation on esophageal and gastric mucosa. Gastrointest Endosc 2000; 52: 342-5.

22. Hage M, Siersema PD, van Dekken H, et al. 5-aminolevulinic acid photodynamic therapy versus argon plasma coagulation for ablation of Barrett's oesophagus: a randomised trial. Gut 2004; 53: 785-90.

23. Orth K, Stanescu A, Ruck A, Russ D, Beger HG. Photodynamic ablation and argon-plasma coagulation of premalignant and early-stage malignant lesions of the oesophagus--an alternative to surgery?. Der Chirurg; Zeitschrift fur alle Gebiete der operativen Medizen 1999; 70: 431-8.

24. Byrne JP, Armstrong GR, Attwood SE. Restoration of the normal squamous lining in Barrett's esophagus by argon beam plasma coagulation. Am J Gastroenterol 1998; 93: 1810-5.

25. Van Laethem JL, Cremer M, Peny MO, Delhaye M, Deviere J. Eradication of Barrett's mucosa with argon plasma coagulation and acid suppression: immediate and mid term results. Gut 1998; 43: 747-51.

26. Ragunath K, Krasner N, Raman VS, Haqqani MT, Phillips CJ, Cheung I. Endoscopic ablation of dysplastic Barrett's oesophagus comparing argon plasma coagulation and photodynamic therapy: a randomized prospective trial assessing efficacy and cost-effectiveness. Scand J Gastroenterol 2005; 40: 750-8.

27. Pereira-Lima JC, Busnello JV, Saul C, et al. High power setting argon plasma coagulation for the eradication of Barrett's esophagus. Am J Gastroenterol 2000;95:1661-8.

28. Van Laethem JL, Jagodzinski R, Peny MO, Cremer M, Deviere J. Argon plasma coagulation in the treatment of Barrett's high-grade dysplasia and in situ adenocarcinoma. Endoscopy 2001; 33: 257-61.

29. Attwood SE, Lewis CJ, Caplin S, Hemming K, Armstrong G. Argon beam plasma coagulation as therapy for high- grade dysplasia in Barrett's esophagus. Clin Gastroenterol Hepatol 2003; 1: 258-63.

30. May A, Gossner L, Gunter E, Stolte M, Ell C. Local treatment of early cancer in short Barrett's esophagus by means of argon plasma coagulation: initial experience. Endoscopy 1999; 31: 497-500.

31. Nomura T, Yamashita K, Miyashita M, Tajiri T. Argon plasma coagulation in Barrett's esophagus. Nihon Rinsho 2005; 63: 1458-62.

32. Pedrazzani C, Catalano F, Festini M, et al. Endoscopic ablation of Barrett's esophagus using high power setting argon plasma coagulation: a prospective study. World J Gastroenterol 2005; 11: 1872-5.

33. Madisch A, Miehlke S, Bayerdorffer E, et al. Long-term follow-up after complete ablation of Barrett's esophagus with argon plasma coagulation. World J Gastroenterol 2005; 11: 1182-6.

34. Schulz H, Miehlke S, Antos D, et al. Ablation of Barrett's epithelium by endoscopic argon plasma coagulation in combination with high-dose omeprazole. Gastrointest Endosc 2000; 51: 659-63.

35. Morris CD, Byrne JP, Armstrong GR, Attwood SE. Prevention of the neoplastic progression of Barrett's oesophagus by endoscopic argon beam plasma ablation. Br J Surg 2001; 88: 1357-62.

36. Kelty CJ, Ackroyd R, Brown NJ, Stephenson TJ, Stoddard CJ, Reed MW. Endoscopic ablation of Barrett's oesophagus: a randomized-controlled trial of photodynamic therapy vs. argon plasma coagulation. Aliment Pharmacol Ther 2004; 20: 1289-96.

37. Grade AJ, Shah IA, Medlin SM, Ramirez FC. The efficacy and safety of argon plasma coagulation therapy in Barrett's esophagus. Gastrointest Endosc 1999; 50: 18-22.

38. Bright T, Watson DI, Tam W, et al. Prospective randomized trial of argon plasma coagulation ablation versus endoscopic surveillance of Barrett's esophagus in patients treated with antisecretory medication. Dig Dis Sci 2009; 54: 2606-11.

39. Bozymski EM. Argon plasma coagulation for non-dysplastic Barrett's epithelium: a hard act to follow. Am J Gastroenterol 2007; 102: 1128-9; author reply 9-30.

40. Dumot JA, Greenwald BD. Argon plasma coagulation, bipolar cautery, and cryotherapy: $\mathrm{ABC}$ 's of ablative techniques. Endoscopy 2008; 40: 1026-32.

41. Mork H, Al-Taie O, Berlin F, Kraus MR, Scheurlen M. High recurrence rate of Barrett's epithelium during longterm follow-up after argon plasma coagulation. Scand J Gastroenterol 2007; 42: 23-7.

42. Sharma P, Wani S, Weston AP, et al. A randomised controlled trial of ablation of Barrett's oesophagus with multipolar electrocoagulation versus argon plasma coagulation in combination with acid suppression: long term results. Gut 2006; 55: 1233-9.

43. Manner H, May A, Miehlke S, et al. Ablation of nonneoplastic Barrett's mucosa using argon plasma coagulation with concomitant esomeprazole therapy (APBANEX): a pros- 
pective multicenter evaluation. Am J Gastroenterol 2006; 101: 1762-9.

44. Dulai GS, Jensen DM, Cortina G, Fontana L, Ippoliti A. Randomized trial of argon plasma coagulation vs. multipolar electrocoagulation for ablation of Barrett's esophagus. Gastrointest Endosc 2005; 61: 232-40.

45. Kahaleh M, Van Laethem JL, Nagy N, Cremer M, Deviere J. Long-term follow-up and factors predictive of recurrence in Barrett's esophagus treated by argon plasma coagulation and acid suppression. Endoscopy 2002; 34: 950-5.

46. Pagani M, Granelli P, Chella B, Antoniazzi L, Bonavina L, Peracchia A. Barrett's esophagus: combined treatment using argon plasma coagulation and laparoscopic antireflux surgery. Dis Esophagus 2003; 16: 279-83.

47. Formentini A, Schwarz A, Straeter J, Stanescu A, HenneBruns D. Treatment of Barrett's esophagus with argon plasma coagulation and antireflux surgery. A retrospective analysis. Hepatogastroenterology 2007; 54: 1991-6.

48. Pinotti AC, Cecconello I, Filho FM, Sakai P, GamaRodrigues JJ, Pinotti HW. Endoscopic ablation of Barrett's esophagus using argon plasma coagulation: a prospective study after fundoplication. Dis Esophagus 2004; 17: 243-6.

49. Ackroyd R, Tam W, Schoeman M, Devitt PG, Watson DI. Prospective randomized controlled trial of argon plasma coagulation ablation vs. endoscopic surveillance of patients with Barrett's esophagus after antireflux surgery. Gastrointest Endosc 2004; 59: 1-7.

50. Morino M, Rebecchi F, Giaccone C, Taraglio S, Sidoli L, Ferraris R. Endoscopic ablation of Barrett's esophagus using argon plasma coagulation (APC) following surgical laparoscopic fundoplication. Surg Endosc 2003; 17: 539-42.

51. Tigges H, Fuchs KH, Maroske J, et al. Combination of endoscopic argon plasma coagulation and antireflux surgery for treatment of Barrett's esophagus. J Gastrointest Surg 2001; 5: 251-9.

52. Ferraris R, Fracchia M, Foti M, et al. Barrett's oesophagus: long-term follow-up after complete ablation with argon plasma coagulation and the factors that determine its recurrence. Aliment Pharmacol Ther 2007; 25: 835-40.

53. Hage M, Siersema PD, Vissers KJ, et al. Molecular evaluation of ablative therapy of Barrett's oesophagus. J Pathol 2005; 205: 57-64.

54. Dotti VP, Baretta GA, Yoshii SO, Ivano FH, Ribeiro HD, Matias JE. [Endoscopic argon plasma thermo-coagulation of Barrett's esophagus using different powers: histopathological and post procedure symptons analysis]. Rev Col Bras Cir 2009; 36: 110-7.

55. Dumoulin FL, Terjung B, Neubrand M, Scheurlen C, Fischer HP, Sauerbruch T. Treatment of Barrett's esophagus by endoscopic argon plasma coagulation. Endoscopy 1997; 29: 751-3.

56. Mork H, Barth T, Kreipe HH, et al. Reconstitution of squamous epithelium in Barrett's oesophagus with endoscopic argon plasma coagulation: a prospective study. Scand J Gastroenterol 1998; 33: 1130-4.

57. Maass S, Martin WR, Spiethoff A, Riemann JF. Barrett esophagus with severe dysplasia in argon beam therapy. $\mathrm{Z}$ Gastroenterol 1998; 36: 301-6.
58. Manner H. Argon plasma coagulation therapy. Curr Opin Gastroenterol 2008; 24: 612-6.

59. Bright T, Watson DI, Tam W, et al. Randomized trial of argon plasma coagulation versus endoscopic surveillance for barrett esophagus after antireflux surgery: late results. Ann Surg 2007; 246: 1016-20.

60. Van Laethem JL, Peny MO, Salmon I, Cremer M, Deviere J. Intramucosal adenocarcinoma arising under squamous reepithelialisation of Barrett's oesophagus. Gut 2000; 46: 574-7.

61. Shand A, Dallal H, Palmer K, Ghosh S, MacIntyre M. Adenocarcinoma arising in columnar lined oesophagus following treatment with argon plasma coagulation. Gut 2001; 48: 580-1.

62. van Hillegersberg R, Haringsma J, ten Kate FJ, Tytgat GN, van Lanschot JJ. Invasive carcinoma after endoscopic ablative therapy for high-grade dysplasia in Barrett's oesophagus. Digestive surgery 2003; 20: 440-4.

63. Allende D, Dumot J, Yerian L. Esophageal squamous cell carcinoma arising after endoscopic ablation therapy of Barrett's esophagus with high-grade dysplasia. Report of a case. Dis Esophagus 2013; 26: 314-8.

64. Smythe A, Elghellal K, Kelty C, Mitton D, Patel K, Ackroyd R. The effect of argon plasma coagulation ablation on esophageal motility and chemoreceptor sensitivity in Barrett's esophagus patients. Dis Esophagus 2010; 23: 445-50.

65. Basu KK, Talwar V, de Caestecker JS. Effects of low-power argon plasma coagulation thermoablation of Barrett's epithelium on oesophageal motility. Eur J Gastroenterol Hepatol 2006; 18: 733-7.

66. Inadomi JM, Somsouk M, Madanick RD, Thomas JP, Shaheen NJ. A cost-utility analysis of ablative therapy for Barrett's esophagus. Gastroenterology 2009; 136: 2101-14 e1-6.

67. Boix J, Lorenzo-Zuniga V, de Vega VM, Planas R. Argon plasma coagulation versus endoscopic mucosal resection in patients with dysplastic Barrett's esophagus. Dig Dis Sci 2009; 54: 1808-9.

68. Lorenzo-Zuniga V, Boix J. Endoscopic mucosal resection and mucosa ablation with argon-plasma coagulation in a high-risk patient with Barrett's oesophagus and oesophageal adenocarcinoma. Dig Liver Dis 2006; 38: 713-4.

69. Basu KK, Pick B, Bale R, West KP, de Caestecker JS. Efficacy and one year follow up of argon plasma coagulation therapy for ablation of Barrett's oesophagus: factors determining persistence and recurrence of Barrett's epithelium. Gut 2002; 51: 776-80.

70. Claydon PE, Ackroyd R. Argon plasma coagulation ablation of Barrett's oesophagus. Scand J Gastroenterol 2005; 40: 617-28.

71. Dijckmeester WA, Wijnhoven BP, Watson DI, et al. MicroRNA-143 and -205 expression in neosquamous esophageal epithelium following Argon plasma ablation of Barrett's esophagus. J Gastrointest Surg 2009; 13: 846-53.

72. Lopes CV, Pereira-Lima J, Hartmann AA. p53 immunohistochemical expression in Barrett's esophagus before and after endoscopic ablation by argon plasma coagulation. Scand J Gastroenterol 2005; 40: 259-63. 